Revue d'histoire de l'Amérique française

REVUE D.HISTOIRE DE L'AMÉRIQUE FRANÇAISE

\title{
L’itinéraire du Frère Marie-Victorin, é.c. (1885-1944)
}

\section{Yves Gingras}

Volume 39, numéro 1, été 1985

URI : https://id.erudit.org/iderudit/304328ar

DOI : https://doi.org/10.7202/304328ar

Aller au sommaire du numéro

Éditeur(s)

Institut d'histoire de l'Amérique française

ISSN

0035-2357 (imprimé)

1492-1383 (numérique)

Découvrir la revue

Citer cette note

Gingras, Y. (1985). L’itinéraire du Frère Marie-Victorin, é.c. (1885-1944). Revue d'histoire de l'Amérique française, 39(1), 77-82. https://doi.org/10.7202/304328ar d'utilisation que vous pouvez consulter en ligne.

https://apropos.erudit.org/fr/usagers/politique-dutilisation/ 
NOTES CRITIQUES

\section{L'ITINÉRAIRE DU FRERE MARIE-VICTORIN, É. C.(1885-1944)}

YVES GINGRAS

Department of History of Science

Harvard University

L'année 1985 marque le centenaire de la naissance du Frère MarieVictorin, des Écoles chrétiennes. La publication récente du volume de Madeleine Lavallée, intitulé Marie-Victorin, un itinéraire exceptionnel ${ }^{1}$, nous fournit l'occasion de rappeler les principales étapes de la carrière de l'auteur de la Flore laurentienne de même que les quelques travaux qui lui ont été consacrés.

Fils d'une famille à l'aise de Québec, Conrad Kirouak voit le jour le 3 avril 1885. Le futur fondateur du Jardin botanique de Montréal a une enfance plutôt paisible mais marquée par une santé fragile (affection pulmonaire) qui le destine très tôt au grand air. Au cours de ses études à l'Académie commerciale de Québec, il se découvre une vocation d'éducateur et veut suivre l'exemple de ses maîtres, les Frères des Écoles chrétiennes. A l'âge de seize ans il entre au noviciat des Frères, le Mont-de-La-Salle à Montréal. Après deux années d'apprentissage il commence à enseigner au niveau primaire au Collège Saint-Jérôme. Suite à un bref passage au Collège Saint-Léon de Westmount, il est rattaché à celui de Longueuil où il enseignera jusqu'en 1920 .

Dès le début de sa carrière d'enseignant il s'initie à la botanique et multiplie les excursions d'herborisation qui le mènent à sa première publication, en 1908, dans Le Naturaliste canadien: «Additions à la flore d'Amérique». A ce point de son apprentissage, la consultation de la Flore canadienne, publiée par l'abbé Léon Provancher en 1862, ne suffit plus et il entre en contact avec plusieurs botanistes nord-américains, dont un professeur de l'Université Harvard, Merritt L. Fernald (spécialiste de la flore de l'Est du Québec), qui l'aide à sortir définitivement de l'amateurisme. Nationaliste, il fonde un cercle de l'ACJC en 1906 où, en jouant des pièces de théâtre (dont certaines composées par Marie-Victorin), les élèves développent leurs sentiments patriotiques.

\footnotetext{
1 Madeleine Lavallée, Marie-Victorin, un itinéraire exceptionnel (Montréal, Éditions Héritage plus, 1984), $272 \mathrm{p}$.
} 
Jusqu'en 1920, on peut dire que Marie-Victorin s'intéresse autant à la nature qu'à la littérature. Le mariage de ces deux activités donne d'ailleurs naissance à deux volumes publiés respectivement en 1919 et 1920, les Récits laurentiens et les Croquis laurentiens, tous deux illustrés par Edmond-J. Massicotte. Quelques années auparavant, en 1916, il publiait son premier ouvrage scientifique, La flore du Témiscouata. Les deux activités lui paraîssent complémentaires et il reproche d'ailleurs vivement à nos écrivains leur ignorance de la flore du Québec. Ainsi, dans un article publié en 1917, il écrit:

Ouvrez n'importe quel recueil de vers canadiens et vous êtes sûr de rencontrer, généralement au bout des lignes, les inévitables primevères et les non moins fatales pervenches. Ces deux mots sont harmonieux, commodes et complaisants pour la rime. Malheureusement ici encore, nous avons affaire à des plantes étrangères à notre flore. ${ }^{2}$

Ces critiques font partie d'un plaidoyer plus général en faveur de l'étude des sciences naturelles. Pour Marie-Victorin, l'indifférence envers ces disciplines est «injustifiable... nuisible et a nui en effet au progrès économique dans la province de Québec». Rappelant que la flore québécoise est mieux connue des Américains que des Canadiens français, il ajoute:

N'oublions pas non plus que chaque nouvelle incursion de la science étrangère sur notre sol, si elle ajoute au capital intellectuel de l'humanité, est en quelque sorte irréparable du point de vue national... Les empiétements, les envahissements de l'anglais sur le français dont nous nous plaignons dans le commerce, l'industrie et les services publics, ne sont rien à côté de ceux dont fatalement, et uniquement par notre incurable indifférence, nous sommes affligés sur le terrain scientifique. ${ }^{3}$

Les talents de pamphlétaire du Frère allaient devenir une arme encore plus efficace après 1920. Cette date, qui marque le début d'une nouvelle période dans l'histoire de l'enseignement supérieur au Québec, constitue aussi une nouvelle étape dans la carrière de Marie-Victorin. A partir de ce moment, on peut même dire que sa biographie se confond avec l'histoire du mouvement scientifique des années 1920 et $1930^{4}$.

$\mathrm{Au}$ moment où l'on doit recruter les premiers professeurs de la nouvelle Faculté des Sciences de l'Université de Montréal, le Frère Marie-Victorin, bien que n'ayant aucun diplôme universitaire, est un

\footnotetext{
2 Frère Marie-Victorin, «L'étude des sciences naturelles. Son développement chez les Canadiens français», Revue canadienne, 20,4 (1917): 284.

3 Ibid., 275

4 Pour plus de détails sur cette période, voir Raymond Duchesne, La science et le pouvoir au Québec (Québec, Éditeur officiel, 1978).
} 
choix tout indiqué pour occuper le poste de professeur de botanique. Jusque-là plutôt isolé dans son poste de sous-directeur du Collège de Longueuil, il pourra dorénavant compter sur des disciples et des collègues qui mettront rapidement en branle un véritable mouvement scientifique qui donnera naissance, entre autres, à l'ACFAS en 1923. Dans cette nouvelle conjoncture, Marie-Victorin pourra reprendre et développer le thème de l'importance des sciences naturelles devant les membres de l'ACFAS ou de la Société canadienne d'histoire naturelle (fondée en 1923) qu'il présidera de 1925 à 1940. C'est d'ailleurs de cette tribune qu'il lancera les projets du Jardin botanique de Montréal en 1929 et de l'Institut de Géologie en 1937. Du point de vue scientifique, sa période universitaire est aussi la plus productive et il peut compter sur de nombreux collaborateurs pour préparer ce qu'il considérera comme l'oeuvre de sa vie: la Flore laurentienne, publiée par les Frères des Écoles chrétiennes en 1935.

Son style incisif et ses talents d'organisateur - sans parler du «charisme» que plusieurs de ses contemporains lui reconnaissent - le placent rapidement à la tête du mouvement scientifique, position que son orgueil (plutôt évident) ne dédaigne d'ailleurs nullement. Dès la fin des années 1920, plusieurs articles sont même publiés sur sa personne et sur son oeuvre et il en vient ainsi à être perçu (et à se percevoir) comme l'incarnation du mouvement scientifique ${ }^{5}$. En 1942, soit deux ans avant la mort accidentelle du Frère, survenue le 15 juillet 1944, Louis-Philippe Audet consacre même un volume complet à ses idées pédagogiques ${ }^{6}$. Quelques années plus tard, le «mythe» Marie-Victorin prendra sa forme définitive dans la biographie écrite par Robert Rumilly ${ }^{7}$, ouvrage qui demeure encore aujourd'hui la meilleure source d'informations sur la vie du botaniste.

Aujourd'hui, Madeleine Lavallée nous offre son Marie-Victorin, un itinéraire exceptionnel, produit de sa collaboration à la préparation d'un film sur la vie du personnage (tourné pour Radio-Québec) et que l'on peut ranger, à côté de celui de Rumilly, parmi les hagiographies. Malgré son titre, ce volume ne constitue pas à proprement parler une biographie de Marie-Victorin. Dans une courte préface, Jules Brunel (bras droit du Frère pendant plus de vingt ans) suggère même qu'il s'agit en quelque sorte d'une autobiographie car le volume est construit autour d'un grand nombre des écrits, publiés ou inédits, de MarieVictorin. De notre point de vue, le volume apparaît plutôt comme une suite de commentaires personnels inspirés de ses écrits. L'autèur cite

\footnotetext{
5 A ce propos, voir J.-C. Guédon, «Du bon usage de la vulgarisation: le cas de MarieVictorin», Questions de culture, 1 (1981): 81-111.

6 Louis-Philippe Audet, Marie-Victorin. Ses idées pédagogiques (Québec, Éditions de l'Érable, 1942).

7 Robert Rumilly, Le Frère Marie-Victorin et son temps (Montréal, Frères des Écoles chrétiennes, 1949).
} 
abondamment le journal inédit (intitulé Mon miroir) que le Frère a tenu de 1903 à 1920 et s'intéresse tout particulièrement à sa personnalité et à sa spiritualité. Malheureusement, les différents morceaux choisis par l'auteur sont pour la plupart inintéressants même s'ils visent à nous faire «percevoir chez le frère cet intérêt extrêmement diversifié dont son esprit ouvert et curieux avait depuis toujours favorisé le développement ${ }^{8}$. Dans l'ensemble, l'ouvrage ajoute donc peu à notre connaissance du personnage et est en bonne partie fondé sur ceux de Rumilly et Audet.

Si l'ouvrage de Madeleine Lavallée ne permet pas vraiment au lecteur de saisir l'aspect «exceptionnel» de l'itinéraire de Marie-Victorin, la publication de son journal serait cependant un pas dans cette direction. A l'instar de celui de Lionel Groulx qui vient de paraître, il pourrait en effet nous éclairer sur la période de formation de cet homme qui a joué un rôle de premier plan dans le mouvement scientifique des années 1920 et 1930. Le centenaire de sa naissance constitue d'ailleurs une excellente occasion pour les Frères des Écoles chrétiennes d'entreprendre la publication de ce manuscrit qui, rappelons-le, couvre la période de 1903 à 1920, année où Marie-Victorin devient professeur à l'Université de Montréal. En 1969, à l'occasion du 25e anniversaire de son décès, le Frère Gilles Beaudet, é.c., avait d'ailleurs publié un volume intitulé Confidence et combat ${ }^{9}$ où il réunissait une centaine de lettres écrites par son illustre confrère entre 1922 et 1944 . La publication de Mon miroir serait donc un excellent complément à ce volume.

En attendant la publication du journal de Marie-Victorin, on pourra lire avec profit la biographie que lui a consacrée Rumilly. Pour une analyse plus sociologique de sa position et de son rôle dans le champ intellectuel des années 1920, on peut consulter l'article de F. Descarries-Bélanger, M. Fournier et L. Maheu, «Le Frère Marie-Victorin et les 'petites sciences'»" ${ }^{10}$. Malgré son titre, cette étude ne se limite pas au seul personnage du Frère des Écoles chrétiennes et propose une analyse globale des pratiques de la première génération des scientifiques québécois actifs au cours des années 1920 et 1930.

Si le rôle social de Marie-Victorin est relativement bien connu, on ignore par contre à peu près tout de l'évolution de sa pensée scientifique et de la valeur de ses contributions à la science botanique. Les circonstances de sa «conversion» au darwinisme, par exemple, n'ont pas vraiment été explorées. Les Prix Grandoger de la Société botanique de France et Coincy de l'Académie des Sciences de Paris, attribués à Marie-

\footnotetext{
8 Madeleine Lavallée, Marie-Victorin, un itinéraire exceptionnel (Montréal, Éditions Héritage plus, 1983), 155.

9 Frère Gilles Beaudet, é. c., Confidence et combat (Montréal, Lidec, 1969).

10 F. Descarries-Bélanger, M. Fournier et L. Maheu, «Le Frère Marie-Victorin et les 'petites sciences'», Recherches sociographiques, 20,1 (1979): 7-39.
} 
Victorin en 1932 et 1935 respectivement, donnent à penser que ses contributions à la botanique (notamment à la floristique et à la phytogéographie) vaudraient la peine d'être étudiées dans le contexte global de l'état de cette discipline au cours des années 1920 et 1930.

Enfin, s'il est vrai comme l'écrit Madeleine Lavallée que «les écrits du Frère Marie-Victorin nous dévoilent sa pensée, ses sentiments et ses combats», alors il vaut peut-être la peine de les lire. Bien qu'il n'existe pas d' «oeuvres complètes» publiées à ce jour, les Éditions Paulines ont recueilli un certain nombre de textes de Marie-Victorin sous le titre Pour l'amour du Québec ${ }^{11}$, textes précédés d'une excellente introduction de Hermas Bastien. Outre des écrits que l'on peut qualifier «de combat» comme «La science et notre vie nationale» (1939) ou «L'étude des sciences naturelles. Son développement chez les Canadiens français» (1917) que nous avons déjà cités, on y retrouve des textes de botanique comme «La flore du pays laurentien» (1916) de même qu'un extrait de la préface à la Flore laurentienne. Le côté littéraire de Marie-Victorin y est aussi représenté par des extraits de ses journaux de voyage, des Récits et des Croquis laurentiens et par une étude sur Menaud, maître draveur, ouvrage que Félix-Antoine Savard a d'ailleurs dédié au botaniste qui lui a appris que «les choses de la nature ont un nom en ce pays».

Pour donner une idée de la vigueur des interventions de MarieVictorin (vigueur qui explique en partie qu'il ait pu devenir le symbole de la science au Canada français), citons brièvement le texte de 1931 où il propose une réorganisation de l'enseignement des sciences naturelles à l'université. Critiquant ce qu'il considère comme des «équivalences platoniques avec les grades de l'Université de Paris», il demande que les diplômes soient mieux adaptés à la réalité nord-américaine. Car, écrit-il, un professeur de science ne peut se dispenser «de vivre dans cette ambiance bilingue sous peine de perdre le contact avec les points de croissance» de sa science. A ceux qui le taxent de francophobie il rétorque:

Elle est sans aucun doute franchement stupide la francophonie qui refuse systématiquement le puissant secours des méthodes françaises et les services de maitres français quand ils sont savants véritables occupés à faire avancer la science... Mais elle est puérile et niaise cette francolâtrie qui ne peut voir la science qu'à travers «l'article de Paris», qui, en tout et pour tout, se tourne vers la Mecque parisienne et qui, coûte que coûte, cherche à endosser un vêtement taillé à la mesure d'un autre. ${ }^{12}$

\footnotetext{
11 Pour l'amour du Québec (Montréal, Éditions Paulines, 1972).

12 Marie-Victorin, «Les sciences naturelles dans l'enseignement supérieur», Revue trimestrielle canadienne, 65 (mars 1931): 27-28.
} 
Ce type d'intervention, typique de ses textes «de combat», l'opppose évidemment à la diplomatie d'un Edouard Montpetit, par exemple, et n'est pas sans lui créer certaines inimitiés. Il reste que, aux côtés de Lionel Groulx et Edouard Montpetit, le Frère Marie-Victorin est sans nul doute l'une des figures les plus importantes du Québec intellectuel. Pour cette raison, on devrait profiter de l'occasion que nous offre le centenaire de sa naissance pour retourner à ses écrits, non pas pour ajouter au mythe, mais pour mieux comprendre la diversité des modes d'intervention des intellectuels dans les domaines politiques et sociaux de même que la diversité des formes que le nationalisme a pu prendre dans le Québec de l'entre-deux-guerres. 